Journal of Biomechanical Engineering

\title{
Multibody kinematics optimization for the estimation of upper and lower limb human joint kinematics: a systematized methodological review
}

\author{
Mickaël Begon \\ Département de Kinésiologie, Université de Montréal, Montréal, QC, Canada \\ Centre de Recherche du Centre Hospitalier Universitaire Sainte-Justine, Montréal, QC, \\ Canada \\ 1700 Jacques Tétreault, \\ Laval, QC H7N 0B6, Canada. \\ mickael.begon@umontreal.ca \\ Michael Skipper Andersen \\ Department of Materials and Production, Aalborg University \\ Fibigerstrade 16, DK-9220 Aalborg East, Denmark \\ msa@make.aau.dk

\section{Raphaël Dumas} \\ Univ Lyon, Université Claude Bernard Lyon 1, IFSTTAR, UMR_T9406, LBMC, F69622, \\ Lyon, France \\ 43 Boulevard du 11 novembre 1918 \\ 69622 Villeurbanne Cedex \\ Raphael.dumas@ifsttar.fr
}


Journal of Biomechanical Engineering

\section{ABSTRACT}

Multibody kinematics optimization (MKO) aims to reduce soft tissue artefact (STA) and is a key step in musculoskeletal modeling. The objective of this review was to identify the numerical methods, their validation and performance for the estimation of the human joint kinematics using MKO.

Seventy-four articles were extracted from a systematized search in five databases and cross-referencing. Model-derived kinematics were obtained using either optimization or Kalman filtering to minimize the difference between measured (i.e., by skin markers, electromagnetic or inertial sensors) and model-derived positions and/or orientations. While hinge, universal and spherical joints prevail, advanced models (e.g., parallel and four-bar mechanisms, elastic joint) have been introduced, mainly for the knee and shoulder joints. Models and methods were evaluated using: (i) simulated data based, however, on oversimplified STA and joint models; (ii) reconstruction residual errors, ranging from $4 \mathrm{~mm}$ to $40 \mathrm{~mm}$; (iii) sensitivity analyses which highlighted the effect (up to $36^{\circ}$ and $12 \mathrm{~mm}$ ) of model geometrical parameters, joint models, and computational methods; (iv) comparison with other approaches (i.e., single body optimization and nonoptimized kinematics); (v) repeatability studies that showed low intra- and inter-observer variability; and (vi) validation against ground-truth bone kinematics (with errors between $1^{\circ}$ and $22^{\circ}$ for tibiofemoral rotations and between $3^{\circ}$ and $10^{\circ}$ for glenohumeral rotations). Moreover, MKO was applied to various movements (e.g., walking, running, arm elevation). 
Journal of Biomechanical Engineering

Additional validations, especially for the upper-limb, should be undertaken and we recommend a more systematic approach for the evaluation of MKO. In addition, further model development, scaling and personalization methods are required to better estimate the secondary degrees-of-freedom.

\section{KEYWORDS}

Kinematic chain, joint model, skin markers, optimization, Kalman filter, validation, state-of-the-art

\section{INTRODUCTION}

There is a growing use of multibody kinematics optimization (MKO) to estimate human joint kinematics from stereophotogrammetry or other motion analysis systems, such as electromagnetic sensors or inertial sensors. While innovative approaches are emerging (e.g. markerless, sensorless or single camera) for motion analysis, they are not commonly used in biomechanics because their accuracy in in-situ complex movements has not yet been well established [1]. MKO is a key step in musculoskeletal modeling, but it is also used in kinematic and dynamic analyses of human movement with the aim of compensating for soft tissue artefact (STA).

Referred to as 'global optimization', 'multibody optimization', 'inverse kinematics' or 'motion reconstruction' according to the field of research, the method is referred to as 
Journal of Biomechanical Engineering

MKO in the present study. This diversity of both terminology and research fields may explain the lack of reviews on this topic. Note that two overviews - although not systematic nor systematized - have recently been published regarding kinematic models of the upper [2] and lower [3] limb used in MKO. MKO simultaneously determines the pose of all segments while enforcing the constraints imposed by various joints in a unique optimization process. By contrast, other kinematics optimization methods (e.g., singular value decomposition method [4], solidification procedure [5], point cluster technique [6], optimal common shape technique [7]) consider each segment independently. They are referred to as single-body kinematics optimization (SKO). MKO requires a model of the osteo-articular system as a kinematic chain, i.e. a series of rigid segments connected by joints, allowing a specific number of degrees-of-freedom (DoFs) to the model. Several joint mechanisms have been developed $[2,3]$ and combined in MKO models. An exhaustive list with their rationale, performance and numerical implementation may help orient the future developments.

The main principle of MKO is to minimize the difference between measured and model-derived skin-marker trajectories (or sensor orientations and positions, velocities etc.) which are subject to some rigid body and kinematic constraints. However, several numerical methods can fulfill this one principle. Their relevance depends on the design variables (i.e., inputs of the optimization problem), objective function, constraints, resolution algorithm, initial guess, etc. Typically, both constrained optimization and Kalman filters have equivalently been implemented as MKO. Kalman filters apply a twostage predictor-corrector procedure in which the system and measurement models are 
Journal of Biomechanical Engineering

used to predict the expected progression of the system states, output and error covariance matrix, which are subsequently corrected by the measurement.

The model-derived kinematics largely depend on the modelling choices and joint kinematics accuracy is not well established. The purpose of this systematized review was to identify the numerical methods and evaluate their performance to estimate human upper and lower limb joint kinematics using MKO from commonly used systems (active/passive makers, electromagnetic and inertial sensors). A secondary objective was to identify the different fields of application of MKO.

\section{METHODS}

\subsection{Search strategy}

An electronic search was performed in October 2016 in Embase, Medline, PubMed, Scopus, and Web of Knowledge. Logical expressions for the search included 'optim* or kalman', 'kinemat* or joint', 'subject or human', and 'model* or over*determ*'. The search was performed on the title, keywords and abstract. The reference lists of key studies were also cross-referenced (i.e., articles either cited in or citing these studies) to obtain further articles. All years of publication were considered.

\subsection{Inclusion and exclusion criteria}

Articles retrieved from the search strategy were appraised by one author according to the following inclusion- exclusion criteria. Only studies in English that reported kinematic results were included. Studies published as conference proceedings, 
Journal of Biomechanical Engineering

studies concerning single body or under-constrained optimization, estimation of joint centers or axes, predictive simulation, studies presenting methods whose outcome is not joint or segment angles, studies performing markerless, sensorless or single-camera motion analysis, studies on cadaveric specimens, animals, robots or machines, studies focusing solely on finger, foot, or spine were omitted.

\subsection{Quality assessment}

A customized checklist of 15 questions was developed based on previous reviews in the field of biomechanics [8-10] to assess the quality of the methods proposed by the studies included in the present review (Appendix, Table S1). Each question was rated zero (no information), one (limited details) or two (satisfying description or justification) or indicated non-applicable (this applies only to questions Q5, Q6, Q7, Q8 and Q10 about experimental setup, evaluation and statistics). Each study was independently evaluated by one of three reviewers (no reviewer evaluated articles he co-authored). Conformity was previously obtained using a sample of 20 articles evaluated by all three authors followed by a discussion to understand and mitigate the difference.

\subsection{Information extraction}

To answer the two aforementioned questions (what are the numerical methods? and what is the type of validation of the MKO?), the following themes were listed and each was addressed by one reviewer: design variables and objective function, constraints and model geometric parameters, resolution algorithm and initial guess, evaluation 
Journal of Biomechanical Engineering

strategy, reference data, and degree of agreement. In order to meet the secondary objective, an additional theme was the field the application of the MKO.

\section{RESULTS}

\subsection{Search yield}

The search results were in Embase: 865 articles, Medline: 803, PubMed: 187, Scopus: 940, Web of Knowledge: 688 . After removing duplicates, the number of articles was 1577. After applying the exclusion criteria, 52 articles were selected for the review and 22 additional articles were obtained by cross-referencing. With all our attempts of logical expressions of keywords, the variety of expressions used in the literature for describing the "multibody kinematics optimization" made the search incomplete without cross-referencing.

\subsection{Quality assessment}

The scores of quality ranged from $13(43.3 \%)$ to $30(100 \%)$, with a mean score of 23 (76.7\%). N/A was occasionally indicated for questions Q5, Q6, Q7, Q8 and Q10 for studies not performing any evaluation, evaluating MKO on simulated data only (i.e., no participants involved), or that did not perform statistical analyses. A score of 0 was indicated in 22 articles for the question Q13 about the quality of the stated limitations. Substantial variability was reported in writing style and article structure between journals dealing with different fields such as biomechanics, robotics, ergonomics, computer 
Journal of Biomechanical Engineering

animation, etc. The relevant information may, therefore, sometimes be difficult to retrieve and subject to the reviewer interpretation.

\subsection{Numerical methods}

Two categories of methods were found in the included studies, namely constrained optimization and Kalman filters. In this review, we consider unconstrained optimization as a special case of constrained optimization. For sake of clarity and comparison between the two categories, the references for the design variables and the constraints of the optimization are grouped in Tables 1 and 2.

\subsubsection{Constrained optimization}

As stated in the introduction, the main principle of MKO is to minimize the difference between measured and model-derived skin-marker trajectories (or sensor orientations and position, velocities, etc.). The next subsections describe the design variables, the objective function, the constraints and initial guess of the optimization problem used in MKO.

\subsubsection{Design variables}

In most cases, the design variables optimized by MKO are joint angles (Table 1). As when using Kalman filters (see section 3.3.2), the number of joint angles and the number of DoFs are the same (i.e., generalized coordinates) and no rigid body or kinematic constraints are required except in the case of additional closed loops [11-13]. 
Journal of Biomechanical Engineering

Nevertheless, quaternions and rotations matrices were also proposed for numerical convenience as well as natural coordinates (i.e., Cartesian coordinates of points and components of axes describing the segments). The design variables can also include the model's geometric parameters (i.e., segment lengths, position of joint centers and orientation of joint axes, position of markers embedded in the segments).

Table 1: Design variables of the MKO problems

\begin{tabular}{|c|c|c|c|}
\hline Variables & Occurrence & $\begin{array}{l}\text { Constrained } \\
\text { optimization }\end{array}$ & Kalman filter \\
\hline Generalized coordinates & $20+6$ & {$[12-32]$} & {$[11,18,33-38]$} \\
\hline Velocities & $2+6$ & {$[32,39]$} & {$[11,33-38]$} \\
\hline Accelerations & $2+6$ & {$[32,39]$} & {$[11,33-37]$} \\
\hline Jerk & 1 & & {$[40]$} \\
\hline Rotation matrices & $9+1$ & [41-49] & [37] \\
\hline Natural coordinates & 8 & [50-57] & \\
\hline Quaternions & 5 & {$[32,58-61]$} & [37] \\
\hline Model parameters & $4+3$ & $\begin{array}{l}{[14,62]^{*}} \\
{[16,22]^{\dagger}}\end{array}$ & [33-35] \\
\hline
\end{tabular}

* within a large-scale optimization (one vector that concatenates time-varying coordinates at all sampled instants of time and constant model parameters)

+ two-level optimization

\subsubsection{Objective function}

Unless inertial [59] and magnetic [21] sensors are used, the objective function used in all studies is the sum of the squared distances between measured and modelderived skin-marker positions. One study replaced the measured skin-marker by virtual markers representing the segment coordinate systems obtained by a SKO [31]. In many studies, some marker weights are introduced in the objective function $[14,23,29-32,41$ - 
Journal of Biomechanical Engineering

$43,45,48,50,61]$. The weights are generally defined according to the marker residuals resulting from a first optimization performed without weights or based on reported amount of STA. In two studies $[29,31]$, marker weights were chosen so that every segment had an equal weighting. Another rationale for the estimation of marker weights was introduced by [27 ], considering the projection of the markers onto a selected axis to cancel the marker (and corresponding STA) effect on this DoF.

Alternative objective functions include the sum of the squared errors between measured (i.e., computed from skin markers without optimization) and model-derived joint angles $[12,63]$, the sum of the squared errors between measured (i.e., obtained from inertial sensors) and model-derived attitude vectors [59], or the weighted sum of the squared errors between measured (i.e., obtained from magnetic sensors) and modelderived positions and attitude vectors [21].

Some penalty terms were also appended to the objective function in order to consider 'soft' kinematic constraints, especially for defining deformable ligaments [12, 51, $54,56]$ or an elastic joint $[43,57]$. A unique study also introduced the sum of the length variations of the musculo-tendon elements as a penalty term [14]. Another study penalizes the knee adduction-abduction [16]. Generally speaking, all terms of the objective function can be considered as 'soft' constraints, i.e. the skin-marker positions (or sensors orientation) defining a set of so-called driving constraints to be minimized [24, $50,56,61]$ 
Journal of Biomechanical Engineering

\subsubsection{Constraints}

According to the choice of the design variables, in case they are not independent of one another, some rigid body constraints must be considered such as the norm of the quaternions, the orthogonally of the rotation matrix, and the constant distances and angles between points and axes describing the rigid segments in case of natural coordinates (Table 2). When the design variables are joint angles, their choice implicitly defines the DoFs of kinematic chain in accordance with the Denavit-Hartemberg convention or Euler/Cardan angles. This means that the joint models are typically defined as spherical, universal or hinge. In this case, the joint angles are sometimes bounded in a physiological range. Otherwise, the kinematic constraints must be defined explicitly as it is specifically done for complex joint models such as parallel or four-bar mechanisms. These kinds of joint models typically include constant length of various ligaments. Other kinematic constraints for complex joints include sphere-on-plane, sphere-on-sphere and point-on-ellipsoid contacts. These kinematic constraints for ligaments and articular contacts are used to model the shoulder, knee and ankle joints. Another possibility is to prescribe coupling equations between the DoFs. Additional kinematic constraints can represent closed loops not within the kinematic chain itself, but with accessories like paddles or footrests [11]. All the aforementioned kinematic constraints stand for 'hard' (deterministic) constraints. As previously explained, the definition of 'soft' constraints relies on the introduction of penalty terms in the objective function. This was typically proposed for some ligaments $[12,51,54,56]$, some scapulothoraric and glenohumeral articular contacts $[12,43]$ and closed loops with the environment [11]. 
Journal of Biomechanical Engineering

The absence of kinematic constraints was implemented as a marginal case of MKO equivalent to a SKO. When the model geometrical parameters are part of the design variables, in the same way as the joint angles, they are sometimes constrained and/or bounded within a physiological range.

Table 2: Constraints in the MKO problems

\begin{tabular}{|c|c|c|c|c|c|}
\hline Constraints & & & Occurrence & Constrained optimization & Kalman filter \\
\hline \multirow{14}{*}{$\begin{array}{l}\text { Hard } \\
\text { constraints }\end{array}$} & \multirow{7}{*}{ Joint } & Spherical, universal or hinge & $26+5+$ & $\begin{array}{l}{[13,14,16-19,21-23,25-27} \\
32,48,49,58,60,62,64-71]\end{array}$ & $\begin{array}{l}{[11,18,33,35,} \\
36,40]\end{array}$ \\
\hline & & & $7 \ddagger$ & {$[18,42,43,46,47,58,61]$} & \\
\hline & & Parallel or 4-bar mechanisms & 5 & {$[52,53,55,70,72]$} & \\
\hline & & Sphere-on-plane & 6 & {$[51,52,54-56,72]$} & \\
\hline & & Sphere-on-sphere & 1 & {$[53]$} & \\
\hline & & Point-on-ellipsoid & 3 & {$[12,20,53]$} & \\
\hline & & Norm of quaternion & 2 & {$[32,61]$} & \\
\hline & \multirow[t]{2}{*}{ Rigid body } & Matrix orthogonality & 1 & {$[45]$} & \\
\hline & & Segment distances/angles* & 2 & {$[50,52]$} & \\
\hline & \multirow{5}{*}{ Ligament } & Conoid & 2 & {$[12,20]$} & \\
\hline & & Cruciate & 7 & {$[51,52,54-56,70,72]$} & \\
\hline & & Collateral & 6 & {$[51,52,54-56,72]$} & \\
\hline & & Patellar & 1 & {$[72]$} & \\
\hline & & Tibiocalcaneal and calcaneofibular & 3 & {$[52,70,72]$} & \\
\hline \multirow{6}{*}{$\begin{array}{l}\text { Soft constraints } \\
\text { (in the objective } \\
\text { function) }\end{array}$} & & Norm of quaternion & 1 & & [37] \\
\hline & & Ligament length & 4 & {$[12,51,54,56]$} & \\
\hline & & Elastic joint & $2+1$ & {$[43,57]$} & [37]§ \\
\hline & & Musculo-tendon length & 1 & {$[14]$} & \\
\hline & & Knee abduction & 1 & {$[16]$} & \\
\hline & & Closed-loop with accessories & 1 & [11] & [11] \\
\hline \multirow{2}{*}{ Bounds } & & Joint range & 4 & {$[11,19,26,73]$} & \\
\hline & & Model parameters & 2 & {$[16,62]$} & \\
\hline Equations & Joint & Coupling motion & $12+1$ & $\begin{array}{l}\text { Knee }[40,65,74-80] ; \\
\text { Ankle }[65,74,79]\end{array}$ & Knee [40] \\
\hline No constraints & & & 5 & {$[51,52,54,75,81]$} & \\
\hline
\end{tabular}

† implicit constraints defined through generalized coordinates

‡ explicit constraints (e.g. combined with natural coordinates)

* in case of natural coordinates

$\S$ spherical joint with penalties on the translation

Note: some references can be cited in different categories since the model includes several joints and authors may have implemented different models. 
Journal of Biomechanical Engineering

\subsubsection{Initial guess and model geometric parameters}

The definition of the initial guess of the optimization process is rarely detailed in the reviewed studies, particularly when the design variables are the joint angles. Nevertheless, the solution for each sampled instant of time can be used as the initial guess for the next one [25]. In MKO based on quaternions or rotation matrices, the initial guess at each sampled instant of time is often defined as the kinematics resulting from SKO applied to the skin markers $[44,58]$. MKO based on natural coordinates reported an initial guess of the points and axes describing the segments similarly obtained from the skin marker positions at each sampled instant of time $[52,56,72]$. As these natural coordinates enclose additional information about the segment geometry, the definition of the model geometrical parameters is also commonly derived from this initial guess by averaging segment lengths, position and orientations of joint centers and axis over all the sampled instants of time. Alternatively $[11,13,15,17,23,24,27,31,47,48,63]$, optimal joint centers and axes can be defined using functional methods (e.g., SARA and SCoRE algorithms $[82,83])$ from dedicated movements. When segment geometry is part of the design variables $[14,16,22,62]$, the geometry is optimized for the movement of interest. Nevertheless, most of the model geometrical parameters are generally defined using the skin marker positions put on bony landmarks in a static posture $[23,41,42,47,49,69]$. Interestingly, one study compared two static postures and demonstrated that the modelderived kinematics is highly affected by the chosen posture [49]. It is, however, in a static posture that generic models are generally scaled to fit the subject anthropometry. Scaling 
Journal of Biomechanical Engineering

is usually based on segment dimensions [28-31, 39, 84] but more complex transformations (i.e., affine or interpolations) based on skin marker positions can also be used $[12,53,60]$. Other scaling strategies rely on geometric fitting of some skin marker positions in multiple static postures [20]. Finally, scaled, generic models are increasingly replaced by subject-specific models. Most of the time, the personalized bone and joint geometries are obtained from magnetic resonance imaging $[43,70,75,78,85]$, computed tomography $[66,67,80]$ or bi-plane radiography [51]. Personalization of the coupling equations between the knee DoFs [80] and personalization of the patellar ligament length [66] were also implemented using fluoroscopy data.

\subsubsection{Kalman filters}

As in constrained optimization, Kalman filtering has also been applied for movement reconstruction. The original Kalman filter [86] was developed for optimal estimation of the system state for linear dynamical systems subject to zero-mean, Gaussian process and measurement noises. Advanced Kalman filter approaches have subsequently been developed to cope with nonlinear systems [87], such as extended and unscented Kalman filters as well as Kalman smoothing, which is an offline estimation approach, where the measurements are filtered both forward and backwards in time.

\subsubsection{State variables}

In all reviewed papers except [37], the state variables contain the generalized joint coordinates with their number matching the number of DoFs of the system and a 
Journal of Biomechanical Engineering

specified number of time derivatives, typically up to accelerations, but there are also studies which include up to the velocities or up to jerk (Table 1). Additionally, Cerveri et al. [34] introduced the local coordinates of markers and joint centers (and their derivatives) in the state variables to enable their online estimations. When the measurements come from inertial sensors and not 2D or 3D markers, state variables were expressed in different ways. Miezal et al. [37] included the translations and orientation of the inertial sensors, their linear and angular velocities and linear accelerations in the state vector. Alternatively, they included the translation and orientation of the segments plus the angular accelerations in the state vector.

\subsubsection{System models}

The system model (or process model) is constructed from a Taylor series expansion such that the highest order derivatives included in the state variables are considered constant. The noise on the system model is modelled as a zero-mean, Gaussian process noise with a specified covariance. Cerveri et al. [35] described how this can be determined as a function of the dynamic content of the system variables, if known. Alternatively, it can be updated online [33].

\subsubsection{Measurement models}

Depending on the measurements available, different measurement models have been developed. When skin marker trajectories are measured, the measurement model expresses the relationship between the state variables and the measured marker 
Journal of Biomechanical Engineering

coordinates, which can either be in the 2D camera image reference frame [33-35] or the 3D laboratory reference frame [40]. As a part of the measurement model, the geometry of the assumed underlying model must be included. This involves the determination of the joint centers and local marker coordinates in technical reference frames. Cerveri et al. [33-35] accomplished this using a standing reference trial and anthropometric measurements, as it is typically accomplished in clinical marker-based analyses. Besides a reference trial, Jackson et al. [18] performed functional trials from which joint centers were determined. Contrary, De Groote et al. [40] used a cadaver-based musculoskeletal model as the template and scaled it linearly to the dimensions of the subject and hereby differences in joint alignment between the subject and the cadaver were not accounted for. In the cases where inertial sensors are used [38] to measure the movements, the measurement model relates the state variables to the measurements from the available sensors such as gyroscopes, accelerometers, magnetometers etc. The measurement model can also be used to enforce constraints which are required for the formulations that do not use generalized joint coordinates in the state vector [37] or which include closed-loop [11]. This is the typical way to implement penalty-based methods (i.e., 'soft' constraints) in Kalman filters.

The measurement noise is modelled as a zero-mean, Gaussian noise with an assumed covariance and is used to specify how confident the user is in the filter prediction compared to the measurements. Choices for the parameters are included in some of the reviewed papers [33-36], but the sensitivity of the predictions to these parameters have not yet been reported. 
Journal of Biomechanical Engineering

\subsubsection{Initial condition}

In the Kalman filters, an initial values of the state vector and an initial estimate of the state covariance matrix are required. Contrary to the constrained optimization approaches, that require an initial guess of the variables at each instant of time and use these to search for an optimal solution, the initial conditions in the Kalman filter specify the solution at the first frame. Subsequent frames evolve from this frame and ideally converge to the expected state estimates within a short time period. As a result, the estimated time evolution of the state variables depends directly on this initial condition. However, the initial condition was only clearly defined in 4 of the 10 papers involving Kalman filtering $[11,35-37]$. In all these, the pose was determined in a starting configuration and all required time derivatives were assumed to be zero. The initial guess of the state covariance matrix was not detailed in any of the reviewed papers.

\subsection{Evaluation and validation of the model-derived kinematics}

Almost all studies, except four, include an evaluation of the optimized kinematic results. However, the results were evaluated using different strategies. The evaluation strategies include assessments using simulated data, reconstruction error, sensitivity analyses, repeatability studies, comparison between various approaches or algorithms and validation against reference data (Table 3). 
Journal of Biomechanical Engineering

Table 3: Types of validation of the MKO. Subcategories are explained in the notes $(*, \dagger, \ddagger)$

\begin{tabular}{|c|c|c|c|}
\hline Type of validation & & Occurence & $\begin{array}{l}\text { Constrained optimization } \\
\text { and Kalman filter }\end{array}$ \\
\hline Simulated data & & 8 & {$[22,33,37,40,44,45,48,50]$} \\
\hline \multirow[t]{2}{*}{ Residual error } & 3D positions & 13 & $\begin{array}{l}{[11,13-16,21,24,32,35,47,50,61,} \\
64]\end{array}$ \\
\hline & 2D positions & 2 & {$[33,35]$} \\
\hline \multirow{3}{*}{$\begin{array}{l}\text { Sensitivity } \\
\text { analysis }\end{array}$} & Monte Carlo & & {$[60,67-69,72,77,85]$} \\
\hline & Geometrical parameters* & 7 & {$[13,17,20,60,63] ;[78,80]$} \\
\hline & Joint constraints & 6 & {$[13,41,46,52,56,79]$} \\
\hline \multirow[t]{2}{*}{ Comparison } & MKO vs SKO and no optimization & 10 & {$[8,23,26,31,34,45,46,61,71,74]$} \\
\hline & Constrained optim. vs Kalman filter & 2 & {$[11,40]$} \\
\hline \multirow{2}{*}{ Repeatability } & Intra-subject & 2 & {$[16,29]$} \\
\hline & Inter-observer & 1 & [16] \\
\hline \multirow{3}{*}{$\begin{array}{l}\text { Validation against } \\
\text { reference data }\end{array}$} & Optoelectronic systems ${ }^{\dagger}$ & 5 & {$[36-38,59,88]$} \\
\hline & Palpation & 2 & {$[12,53]$} \\
\hline & Bone kinematics & 12 & $\begin{array}{l}{[27,39,54,81] \underline{50} ;[43,49,57,66,76]} \\
{[51,55]}\end{array}$ \\
\hline None & & 4 & {$[19,25,42,73]$} \\
\hline
\end{tabular}

Notes: the references are organized as follow:

* regression versus functional methods; generic versus personalized

$\dagger$ in case of inertial and magnetic measurement units

‡ intracortical pins; dynamic stereo-radiography; and biplanar X-rays

\subsubsection{Evaluation using simulated data}

Some innovative algorithms for MKO were initially tested using simulated data, often as a proof of concept (e.g., convergence and calculation cost), where noise is added to the reference kinematics. This noise, modelling the STA, was almost always defined as random and continuous components initially proposed by [5]. A summary of noise parameters for the lower limb can be found in [28]. Generally, MKO showed a good ability to cope with this simulated noisy data, highlighting the relevance of innovative algorithms or their superiority over conventional methods (see section 3.4.4). 
Journal of Biomechanical Engineering

\subsubsection{Reconstruction error}

Nearly a quarter of the experimental studies reported the mean residual error on the marker positions. This metric derives from the objective function and was used to compare different models and methods, marker sets or levels of personalization. Similarly, Cerveri et al. $[33,35]$ also reported the reconstruction error in pixels relative to the marker 2D position in each camera image reference frame. In essence, a large range of residual values were found $(4-40 \mathrm{~mm})$ according to the complexity of the kinematic chain model (e.g., upper-limb or full body), the degree of personalization and the movement of interest.

\subsubsection{Sensitivity analyses}

Most sensitivity analyses focused on uncertainties of continuous variables (e.g., segment length, position of joint center) and used Monte Carlo methods. Different sets of geometrical parameters obtained by regression versus functional methods or scaled generic versus personalized models were also compared. Additionally, the effect of different joint constraints was widely assessed.

Although each study used different ranges of uncertainty and different metrics, resulting standard deviation in model-derived joint kinematics reached up to $36^{\circ}$ and 12 $\mathrm{mm}$ [72]. Sensitivity analyses demonstrated that STA had the most deleterious effect on hip gait kinematics $[28,77]$, while errors in bony landmark location resulted in moderate effects $[69,72,77,85]$. Moreover, sensitivity of MKO to reduced marker sets $[15,64]$ 
Journal of Biomechanical Engineering

showed good performance with less than three markers per body segment. Yet, MKO cannot limit the discrepancy attributed to different marker sets [29].

In terms of model geometry, functionally located centers of rotation for the hip, knee, and ankle gave slightly modified joint angles [63], while functionally determined axes and centers of rotation on the upper-limb resulted in larger pronation-supination range of motion $[13,17]$. Prinold and Bull $[20]$ showed that shoulder models without appropriate scaling of the ellipsoid resulted in kinematics spikes during pull-ups.

Joint constraints affect the resulting joint kinematics, especially the secondary (also called combined) DoFs like knee abduction or rotation [41, 46, 52, 79]. Joint constraints also affect the location of the knee lateral and medial contact points [56].

\subsubsection{Comparison between approaches or algorithms}

Multiple comparative studies were carried out between MKO, SKO and joint kinematics estimated without optimization. Only two studies compared constrained optimization and Kalman filtering. Some features favoring MKO over SKO or nonoptimized kinematics were inter and intra-observer repeatability [16], robustness to marker mislocation [71], and avoidance of joint dislocations [45, 61, 79]. Most studies reinforced the conclusion that the effect of joint constraints is more pronounced on the secondary DoFs of gait $[23,46,74]$. Comparisons between constrained optimization and Kalman filtering showed that the benefit of Kalman filtering is not visible on the joint angle time histories but, unsurprisingly, on the velocities and accelerations $[11,40]$. 
Journal of Biomechanical Engineering

\subsubsection{Repeatability studies}

Only two studies reported the repeatability of the MKO approach $[16,29]$. MKO showed a lower intra-subject and inter-observer variability than the commonly used Newington-Helen Hayes gait model for clinically significant output variables (joint angles, forces and moments) [16]. Moreover, using MKO, the inter-trial variability was consistent across different marker sets [29].

\subsubsection{Validation against reference data}

Only 12 of the 74 studies validated the optimized kinematics against a gold standard, which consists of ground-truth bone kinematics of upper or lower-limbs obtained using either intracortical pins, dynamic stereo-radiography, and biplanar X-rays. Interestingly, the study of Seth et al. [39] is the only one that did not assess the ability of MKO to reduce STA but rather the bio-fidelity of the model, i.e. its capacity to replicate errorless skeletal kinematics.

When validated against reference data on asymptomatic subjects, the typical errors for the model-derived tibiofemoral rotations were between $1^{\circ}$ and $22^{\circ}$ and between $1 \mathrm{~mm}$ and $8 \mathrm{~mm}$ during squat, stair ascent, gait and running movements and the errors were maximal for internal-external rotation, anterior-posterior and proximal-distal displacements $[51,54,55,57,81]$. A detailed outline of the errors for the model-derived tibiofemoral kinematics can be found in [3]. Typical errors for the model-derived glenohumeral rotations were between $3^{\circ}$ and $10^{\circ}$ during arm flexion and abduction and the errors were maximal for internal-external rotation [27, 43]. Studies have highlighted 
Journal of Biomechanical Engineering

that detailed (i.e., including ligament constraints) $[54,55]$ and subject-specific $[51,66]$ knee joint models are required to accurately estimate the secondary knee DoF kinematics. Indeed, hinge or spherical joint models led to larger errors than non-optimized kinematics or double calibration $[49,81]$.

In terms of technologies, comparisons were performed between inertial and magnetic measurement units and optoelectronic systems (although not a 'gold standard', authors consider this as the method of reference). For the upper limb, especially for the scapula, two studies compared the model-derived kinematics with manual palpation (again, here, considered as a reference method).

\subsection{Fields of application}

MKO applications include clinical and sports biomechanics (Table 4). Gait, especially walking in a straight line but also with changes in direction, have been the most studied movements ( $50 \%$ of the studies). Other activities of daily living involving the lower extremities like stair descent/ascent, sit-to-stand motion, manual wheelchair transfer, and generic reach and steering movements involving the upper limb were also of interest. Analytical movements of the upper-limb (arm elevation and rotation as well as elbow flexion and pronation-supination) were studied. Studies including analytical movements of the lower-limb were less frequent. Regarding sports biomechanics, several movements were assessed, namely running, jumping, side-cutting maneuvers, squats and, in an isolated manner, pull-up, movements on a slideboard, in gymnastics, fencing, and kayak. 
Journal of Biomechanical Engineering

Table 4: Fields of MKO applications. Subcategories are explained in the notes $\left(*,+, \ddagger, \S,{ }^{\prime}, "\right)$

\begin{tabular}{|c|c|c|c|}
\hline Activities & & Occurrence & Constrained optimization and Kalman filter \\
\hline \multirow[t]{3}{*}{$\begin{array}{l}\text { Daily living } \\
\text { activities }\end{array}$} & Lower-Limb : Walking* & 37 & $\begin{array}{l}{[16,22,25,28-31,33,35,38,40-42,45-47,52,56,59-} \\
62,67-72,74,75,77-81,85,88] ;[66]\end{array}$ \\
\hline & Lower-Limb: others ${ }^{\dagger}$ & 7 & {$[46,49,57,58,70] ;[49,70]$} \\
\hline & Upper-limb & 4 & {$[64] ;[35,50] ;[50]$} \\
\hline \multirow{2}{*}{$\begin{array}{l}\text { Analytical } \\
\text { movements }\end{array}$} & Lower-limb & 4 & {$[22,34,49,62]$} \\
\hline & Upper limb§ & 14 & {$[12,18,20,21,27,43,48,53] ;[13,17,19,36,48,73]$} \\
\hline \multirow[t]{3}{*}{ Sports activities } & Lower-limb' & 10 & {$[54,76] ;[24,58,84] ;[23] ;[51,55,59,65]$} \\
\hline & Upper-limb (pull-up) & 1 & [20] \\
\hline & Whole body" & 4 & [59]; [15]; [33]; [11] \\
\hline
\end{tabular}

Notes: the references are organized as follow:

* Straight walking; Walking with change in direction

+ Stair descent/ascent; Sit-to-stand

‡ Manual wheelchair transfer; Generic reach; Steering movements

$\S$ Arm elevation and rotation; Elbow flexion and pronation-supination

' Running; Jumping; Side-cutting maneuvers; Squats

" Slideboard; Gymnastics; Fencing; Kayak

While the main objective of the MKO has been to obtain accurate joint kinematics by reducing the STA, a unique group of researchers focused on the dissociation of the bone and soft tissue kinematics to estimate the movement of the wobbling mass [24]. These authors state that since the accelerations of the bones and soft tissues are different, it influences the joint dynamics $[89,90]$. Moreover, some studies explored other benefits like the ability to use a reduced marker set $[15,64]$. Indeed, compared to the methods which model segments independently (i.e., six DoFs each), the joint constraints reduce the number of DoF number in the model. Consequently, fewer markers are required to estimate the joint kinematics. Limited marker sets may be useful when it is anticipated that markers will be occluded by the movement itself (e.g., body in tuck or pike postures). 
Journal of Biomechanical Engineering

Only eight studies evaluated the performance of MKO on a pathological population, namely patients with knee osteoarthritis $[51,55]$, knee ligament deficiency $[76,80]$, knee prosthesis $[49,66]$, and cerebral palsy $[75,78]$. Pregnant $[41,42]$, postmenopausal women [67] and elderly [46] were also studied.

\section{DISCUSSION}

In light of the emerging development and application of MKO algorithms, this article aimed at reviewing the MKO methods and models as well as their validation for estimating upper and lower limb joint kinematics, and their fields of application in terms of activities and outcomes, leading to the following synthesis.

\subsection{Methods and models}

\subsubsection{Implementation}

Constrained optimization and Kalman filters can be equivalently implemented as MKO to obtain model-derived joint kinematics. Without joint constraints, MKO is equivalent to SKO and Kalman filters have also already been used in this case (e.g., [91]). However, constrained optimization seems to be more suitable for complex joint models, and closed loops. The choice of the design variables, generalized or natural coordinates, is based on numerical convenience and many have been proposed in the literature. Generalized coordinates (joint angles) are commonly used with hinge or spherical joints and natural coordinates with more advanced joint models such as parallel mechanisms. 
Journal of Biomechanical Engineering

Still, velocities and accelerations are rarely included in the state variables $[32,39]$, although their inclusion ensures that a dynamically consistent solution can be found, which is essential for dynamics.

In addition, with few exceptions $[25,30]$, constrained optimization is generally performed off-line. Conversely, Kalman filtering is typically a real-time method except in the case of Kalman smoothing [40]. The implementation of constraints in Kalman filters is not trivial unless done through penalty-based methods $[11,37]$. Because the state variables include the velocities, accelerations and sometimes the jerks in addition to the generalized coordinates, the Kalman filter provides joint kinematics without discontinuities by integrating the time domain. Nevertheless, many gains (i.e., system and measurement covariance matrices) must be tuned to avoid divergence.

\subsubsection{Joint models}

One important issue in the MKO is the definition of the joint models. This section summarizes both constraints in the constrained optimization and state variables in the Kalman filters. Other literature reviews, although not systematic nor systematized reviews, have been published on this particular topic where additional upper limb joint models have been listed [2, 92, 93]. In addition, a historical perspective for the introduction of the different lower limb joint models as well as their anatomical significance have been presented [3]. 
Journal of Biomechanical Engineering

\subsubsection{Upper limb}

The types of joints adopted within upper limb modelling generally include an open-loop kinematic chain including universal sternoclavicular, elbow and wrist joints and a spherical glenohumeral joint $[17,19,21,33,64]$. In this case, no acromioclavicular joint was modelled, with only a clavicle segment linking the thorax and humerus. Alternatively, sternoclavicular and acromioclavicular joints were modelled as spherical $[13,18,27,43]$. Elbow and wrist joints were modelled as hinge and spherical, respectively [14, 35] or both spherical $[48,58,65]$. Additionally, to allow for glenohumeral translations, two studies considered six DoFs $[25,48]$, while another considered translations as 'soft' constraints [43]. Conversely, closed loops with a scapulothoracic (point(s)-on-ellipsoid) joint [12, 20, 53], and with humeroradial (spherical), humeroulnar (linear annular) and radioulnar (spherical) joints [13] were also proposed. Other joint models relied on the definition of scapular and clavicular rhythms $[39,65]$.

\subsubsection{Lower limb}

The types of joints selected for lower limb modelling generally include spherical hip, hinge knee and universal ankle joints $[11,22,25,32,42,60,62,66-69]$. The universal joint at the ankle was either modelled as concurrent or, more physiologically, nonconcurrent axes to account for both talo-crural and subtalar joints $[22,25,40,60,62,66$, $69,70]$. Alternatively, spherical hip, knee and ankle joints have been also widely used in MKO $[16,23,46,47,49,58,61,71]$, where only the translations were constrained. A 
Journal of Biomechanical Engineering

unique study modelled the knee joint as universal [26] while another considered six Dofs [31]. Parallel mechanisms or four-bar mechanisms were also proposed as more realistic models for the knee $[52,55,70,72]$ and ankle joints $[52,70,72]$, seldom with deformable ligaments $[51,54,56]$. Few studies modelled the knee joint with both tibiofemoral and patelloferomal mechanisms $[60,66,72]$. Other models of knee $[40,65,74-80]$ and ankle joints $[65,74,79]$ relied on the definition of coupling equations between the flexionextension and the other DoFs. 'Soft' constraints that defined deformable ligaments with a penalty-based method were also proposed $[51,54,56]$. The minimization of the elastic energy defined by the knee joint stiffness matrix was another penalty implementation [57].

\subsection{Validation and performance}

The performance of MKO has been assessed using various approaches that can be sorted in six categories of relevance:

i) Algorithm reliability using simulated data: The main aim of MKO is to reduce, using a priori knowledge of joint mechanisms, the rigid component of the STA (i.e., the rotation and translation of the marker-cluster relative to the bone). This rigid component is the main cause of the joint dislocations obtained in the estimated kinematics $[94,95]$. Consequently, we recommend including more realistic STA models (e.g., $[96,97])$ when the algorithm reliability is assessed using simulated data, as recently performed by [28]. Also, simulated marker 
Journal of Biomechanical Engineering

trajectories that derive from models with simplified joint mechanisms, cannot fully illustrate the benefit of MKO over SKO or non-optimized kinematics.

ii) Residual errors of marker positions: As discussed by some authors (e.g., [61]), marker residuals may represent the amount of STA but also the adequacy between the kinematic chain (i.e., number of DoFs and geometrical parameters) and the skeleton. Nevertheless, reducing the number of DoFs, e.g., by defining closed-loops $[13,39]$, without increasing the marker residual may indicate a higher biofidelity of the model. Marker residuals should not be considered as a validation but we recommend providing the range of values with comparison to reported STA range [10].

iii) Sensitivity analysis: Sensitivity analyses are essential to evaluate how uncertainties propagate to joint kinematics as well as to determine the geometrical parameters that most affect the model output. These parameters are typically the ones to be personalized. While geometrical parameters may be defined based on medical images $[51,68,70,75,78]$, this approach is timeconsuming and costly for clinical application. Moreover, descriptive anatomy is not always consistent with functional anatomy, especially for complex joints [98]. Development of techniques for non-homogenous scaling [12, 20, 60, 65] and parameters identification before $[17,60]$ or simultaneously with the motion of interest $[14,16,22,62]$, should be continued. Sensitivity analysis on the numerical parameters of the MKO such as the weights of penalty terms in 
Journal of Biomechanical Engineering

constrained optimization [56] and covariance of the process noise in Kalman filters should also be generalized.

iv) Kinematics comparison with various noninvasive methods: Without joint constraints, MKO is equivalent to SKO. Both differ from non-optimized kinematics (sometimes called direct kinematics $[8,29,30]$ although it corresponds to an inverse kinematics approach stricto sensu) which is most affected by STA. Most of the differences in the joint kinematics can be attributed to the introduction of the joint constraints but also to the different definition of anatomical frames that can associated to each method [31, 75]. In the comparative studies, differences are usually expressed in terms of root mean square (RMS) differences. Robinson et al. [23] also compared the time histories of the joint angles using statistical parametric mapping [99]. This statistical approach may be more informative about the phases of the movement with large differences between models. The main limitation of comparative studies comes from the lack of a gold standard. Often, the commonly used approach (e.g., non-optimized kinematics) serves as a reference. However, the objective of MKO is to solve limitations coming from commonly used approaches, such as joint dislocation and changes in segment length.

v) As stated by [75], the repeatability of new model needs to be assessed before being introduced clinically. By constraining the joint kinematics to physiological motions, the feasible region is smaller and the solution less 
Journal of Biomechanical Engineering

sensitive to STA. While Charlton et al. [16] showed that MKO outperforms commonly used models (e.g., Newington-Helen Hayes gait model) in terms of intra-subject and inter-observer repeatability, further studies are required to confirm the clinical relevance of MKO.

vi) Validation against ground-truth bone kinematics: While only 12 studies assessed the MKO accuracy against ground-truth bone kinematics, other approaches (i.e., SKO and non-optimized kinematics) also have not extensively been validated using gold standard measurements. The main reason is the scarcity of datasets including several makers (or sensors) and bone kinematics. A recent initiative to provide open source benchmark data [100] should be commended. To evaluate the degree of agreement between model-derived and measured joint kinematics, Richard et al. [57] used a Bland and Altman analysis [101]. Such statistical approaches of agreement may be more informative than root mean squared errors and coefficient of determination. Since only 3 out of the 12 validation studies deal with the upper-limb, such validations should be multiplied in the future.

\subsection{Beyond kinematics}

In combination with body segment inertial parameters and ground reaction forces, model-derived joint kinematics have been used to calculate joint moments [21, $23,30,40,42,46,61,68,70,77,85]$ and variables that derive from musculoskeletal 
Journal of Biomechanical Engineering

models (e.g., musculo-tendon forces or joint contact forces $[12,14,25,28,60,66,68,70$, $77,80,84,85]$ ) or in combination with finite-element models (e.g. femoral strain [67]). Joint constraints reportedly affected less joint kinetics than kinematics $[46,69]$ in gait, but significant differences were found in side-cutting maneuvers [23] and upper-limb movements [13]. Moreover, STA $[28,77]$ and joint constraints $[70,84]$ had an effect on musculo-tendon and joint contact forces. Kalman filters compared to constrained optimization can lead to more physiological joint moments [40]. While about $17 \%$ of the studies reported musculoskeletal variables, additional $30 \%$ introduced or discussed the need of MKO for musculoskeletal applications. Unless when Kalman filters are used [40] or when wobbling masses are specifically included in the modelling [89], MKO generally has a limited effect on joint moments. The main effect on musculo-tendon and joint contact forces seems to come directly from the number of DoFs of the musculoskeletal model $[70,84,102-104]$ and this has to be taken into account when defining the joint models.

\section{CONCLUSION}

This systematized review has highlighted a variety of MKO algorithms and of joint models, including advanced mechanisms, especially for the knee and shoulder joints. However, the ability of MKO to accurately estimate joint kinematics depends on both

models (e.g., joint constraints, geometrical parameters, etc.) and methods (e.g., weighting, initial condition, etc.) to prevent deleterious effects of STA on the secondary 
Journal of Biomechanical Engineering

degrees-of-freedom, often associated to pathologies. Model personalization requires further developments to better estimate these secondary DoFs. MKO has been assessed in several ways but only 12 out of 74 studies included validation against ground-truth bone kinematics. Additional validations, especially for the upper-limb, should be undertaken.

We recommend a more systematic approach for the evaluation of the MKO algorithms, in agreement with the best practices proposed for musculoskeletal models $[105,106]$ : (i) Assess the biofidelity of the joint model using gold standard data of the movement of interest when possible as in Seth et al. [39]; (ii) Test the robustness of the model and of the method by evaluating the sensitivity of the joint kinematics to both geometrical and numerical parameters and to STA; (iii) Ensure that the marker residuals remain within the STA range reported in the literature [10]; (iv) Compare resulting joint kinematics to those already available in the literature; $(v)$ Blindly validate MKO using datasets with both ground-truth bone and skin marker kinematics as provided in Cereatti et al. [100] when possible; and (vi) Assess the repeatability and reliability of the method for clinical applications.

Finally, we encourage authors to use the expression 'multibody kinematics optimization' to better track its evolution and benefit in future reviews and to avoid confusion with other well-established names in various fields such as 'global optimization' and 'inverse kinematics'. 
Journal of Biomechanical Engineering

\section{Acknowledgement}

The authors acknowledge Denis Arvisais for his help in the database search. 
Journal of Biomechanical Engineering

\section{References}

[1] Schmitz, A., Ye, M., Shapiro, R., Yang, R. G., and Noehren, B., 2014, "Accuracy and repeatability of joint angles measured using a single camera markerless motion capture system," Journal of Biomechanics, 47(2), pp. 587-591.

[2] Duprey, S., Naaim, A., Moissenet, F., Begon, M., and Cheze, L., 2016, "Kinematic models of the upper limb joints for multibody kinematics optimisation: An overview," Journal of Biomechanics.

[3] Leardini, A., Belvedere, C., Nardini, F., Sancisi, N., Conconi, M., and Parenti-Castelli, V., 2017, "Kinematic models of lower limb joints for musculo-skeletal modelling and optimization in gait analysis," Journal of Biomechanics.

[4] Soderkvist, I., and Wedin, P. A., 1993, "Determining the movements of the skeleton using well-configured markers," Journal of Biomechanics, 26(12), pp. 1473-1477.

[5] Cheze, L., Fregly, B. J., and Dimnet, J., 1995, "A solidification procedure to facilitate kinematic analyses based on video system data," Journal of Biomechanics, 28(7), pp. 879-884.

[6] Andriacchi, T. P., Alexander, E. J., Toney, M. K., Dyrby, C., and Sum, J., 1998, "A point cluster method for in vivo motion analysis: applied to a study of knee kinematics," Journal of Biomechanical Engineering, 120(6), pp. 743-749.

[7] Taylor, W. R., Ehrig, R. M., Duda, G. N., Schell, H., Seebeck, P., and Heller, M. O., 2005, "On the influence of soft tissue coverage in the determination of bone kinematics using skin markers," Journal of Orthopaedic Research, 23(4), pp. 726-734.

[8] Kainz, H., Carty, C. P., Modenese, L., Boyd, R. N., and Lloyd, D. G., 2015, "Estimation of the hip joint centre in human motion analysis: A systematic review," Clinical Biomechanics, 30(4), pp. 319-329.

[9] Lempereur, M., Brochard, S., Leboeuf, F., and Rémy-Néris, O., 2014, "Validity and reliability of 3D marker based scapular motion analysis: A systematic review," Journal of Biomechanics, 47(10), pp. 2219-2230.

[10] Peters, A., Galna, B., Sangeux, M., Morris, M., and Baker, R., 2010, "Quantification of soft tissue artifact in lower limb human motion analysis: A systematic review," Gait \& Posture, 31(1), pp. 1-8.

[11] Fohanno, V., Begon, M., Lacouture, P., and Colloud, F., 2014, "Estimating joint kinematics of a whole body chain model with closed-loop constraints," Multibody System Dynamics, 31(4), pp. 433-449.

[12] Bolsterlee, B., Veeger, H. E. J., and van der Helm, F. C. T., 2014, "Modelling clavicular and scapular kinematics: from measurement to simulation," Medical \& Biological Engineering \& Computing, 52(3), pp. 283-291.

[13] Laitenberger, M., Raison, M., Périé, D., and Begon, M., 2015, "Refinement of the upper limb joint kinematics and dynamics using a subject-specific closed-loop forearm model," Multibody System Dynamics, 33(4), pp. 413-438.

[14] Ayusawa, K., Ikegami, Y., and Nakamura, Y., 2014, "Simultaneous global inverse kinematics and geometric parameter identification of human skeletal model from motion capture data," Mechanism and Machine Theory, 74, pp. 274-284.

[15] Begon, M., Wieber, P.-B., and Yeadon, M. R., 2008, "Kinematics estimation of straddled movements on high bar from a limited number of skin markers using a chain model," Journal of Biomechanics, 41(3), pp. 581-586. 
[16] Charlton, I. W., Tate, P., Smyth, P., and Roren, L., 2004, "Repeatability of an optimised lower body model," Gait \& Posture, 20(2), pp. 213-221.

[17] Fohanno, V., Lacouture, P., and Colloud, F., 2013, "Improvement of upper extremity kinematics estimation using a subject-specific forearm model implemented in a kinematic chain," Journal of Biomechanics, 46(6), pp. 1053-1059.

[18] Jackson, M., Michaud, B., Tétreault, P., and Begon, M., 2012, "Improvements in measuring shoulder joint kinematics," Journal of Biomechanics, 45(12), pp. 2180-2183.

[19] Pontonnier, C., and Dumont, G., 2009, "Inverse dynamics method using optimization techniques for the estimation of muscles forces involved in the elbow motion," International Journal on Interactive Design and Manufacturing (IJIDeM), 3(4), pp. 227-236.

[20] Prinold, J. A. I., and Bull, A. M. J., 2014, "Scaling and kinematics optimisation of the scapula and thorax in upper limb musculoskeletal models," Journal of Biomechanics, 47(11), pp. 2813-2819.

[21] Prokopenko, R. A., Frolov, A. A., Biryukova, E. V., and Roby-Brami, A., 2001 , "Assessment of the accuracy of a human arm model with seven degrees of freedom," Journal of Biomechanics, 34(2), pp. 177-185.

[22] Reinbolt, J. A., Schutte, J. F., Fregly, B. J., Koh, B. I., Haftka, R. T., George, A. D., and Mitchell, K. H., 2005, "Determination of patient-specific multi-joint kinematic models through two-level optimization," Journal of Biomechanics, 38(3), pp. 621-626.

[23] Robinson, M. A., Donnelly, C. J., Tsao, J., and Vanrenterghem, J., 2014, "Impact of knee modeling approach on indicators and classification of anterior cruciate ligament injury risk," Medicine \& Science in Sports \& Exercise, 46(7), pp. 1269-1276.

[24] Thouzé, A., Monnet, T., Bélaise, C., Lacouture, P., and Begon, M., 2016, "A chain kinematic model to assess the movement of lower-limb including wobbling masses," Computer Methods in Biomechanics and Biomedical Engineering, 19(7), pp. 707716.

[25] van den Bogert, A. J., Geijtenbeek, T., Even-Zohar, O., Steenbrink, F., and Hardin, E. C., 2013, "A real-time system for biomechanical analysis of human movement and muscle function," Medical \& Biological Engineering \& Computing, 51(10), pp. 10691077.

[26] Tsai, M.-J., and Lung, H.-Y., 2014, "Two-phase optimized inverse kinematics for motion replication of real human models," Journal of the Chinese Institute of Engineers, 37(7), pp. 899-914.

[27] Begon, M., Bélaise, C., Naaim, A., Lundberg, A., and Chèze, L., 2016, "Multibody kinematic optimization with marker projection improves the accuracy of the humerus rotational kinematics," Journal of Biomechanics.

[28] Lamberto, G., Martelli, S., Cappozzo, A., and Mazzà, C., 2016, "To what extent is joint and muscle mechanics predicted by musculoskeletal models sensitive to soft tissue artefacts?," Journal of Biomechanics.

[29] Mantovani, G., and Lamontagne, M., 2017, "How different marker sets affect joint angles in inverse kinematics framework," Journal of Biomechanical Engineering, 139(4). 
Journal of Biomechanical Engineering

[30] Pizzolato, C., Reggiani, M., Modenese, L., and Lloyd, D. G., 2017, "Realtime inverse kinematics and inverse dynamics for lower limb applications using OpenSim," Computer Methods in Biomechanics and Biomedical Engineering, 20(4), pp. 436-445.

[31] Lathrop, R. L., Chaudhari, A. M. W., and Siston, R. A., 2011, "Comparative assessment of bone pose estimation using point cluster technique and OpenSim," Journal of Biomechanical Engineering, 133(11), p. 114503.

[32] Andersen, M. S., Damsgaard, M., and Rasmussen, J., 2009, "Kinematic analysis of over-determinate biomechanical systems," Computer Methods in Biomechanics and Biomedical Engineering, 12(4), pp. 371-384.

[33] Cerveri, P., Rabuffetti, M., Pedotti, A., and Ferrigno, G., 2003, "Real-time human motion estimation using biomechanical models and non-linear state-space filters," Medical and Biological Engineering and Computing, 41(2), pp. 109-123.

[34] Cerveri, P., Pedotti, A., and Ferrigno, G., 2005, "Kinematical models to reduce the effect of skin artifacts on marker-based human motion estimation," Journal of Biomechanics, 38(11), pp. 2228-2236.

[35] Cerveri, P., Pedotti, A., and Ferrigno, G., 2003, "Robust recovery of human motion from video using Kalman filters and virtual humans," Human Movement Science, 22(3), pp. 377-404.

[36] El-Gohary, M., and McNames, J., 2012, "Shoulder and elbow joint angle tracking with inertial sensors," IEEE Transactions on Biomedical Engineering, 59(9), pp. 2635-2641.

[37] Miezal, M., Taetz, B., and Bleser, G., 2016, "On inertial body tracking in the presence of model calibration errors," Sensors, 16(7).

[38] Zhang, Z. Q., Wong, W. C., and Wu, J. K., 2011, "Ubiquitous human upperlimb motion estimation using wearable sensors," IEEE Transactions on Information Technology in Biomedicine, 15(4), pp. 513-521.

[39] Seth, A., Matias, R., Veloso, A. P., and Delp, S. L., 2016, "A biomechanical model of the scapulothoracic joint to accurately capture scapular kinematics during shoulder movements," PLoS ONE, 11(1), p. e0141028.

[40] De Groote, F., De Laet, T., Jonkers, I., and De Schutter, J., 2008, "Kalman smoothing improves the estimation of joint kinematics and kinetics in marker-based human gait analysis," Journal of Biomechanics, 41(16), pp. 3390-3398.

[41] Aguiar, L., Andrade, C., Branco, M., Santos-Rocha, R., Vieira, F., and Veloso, A., 2016, "Global optimization method applied to the kinematics of gait in pregnant women," Journal of Mechanics in Medicine and Biology, 16(06), p. 1650084.

[42] Aguiar, L., Santos-Rocha, R., Branco, M., Vieira, F., and Veloso, A., 2014, "Biomechanical model for kinetic and kinematic description of gait during second trimester of pregnancy to study the effects of biomechanical load on the musculoskeletal system," Journal of Mechanics in Medicine and Biology, 14(01), p. 1450004.

[43] Charbonnier, C., Chagué, S., Kolo, F. C., Chow, J. C. K., and Lädermann, A., 2014, "A patient-specific measurement technique to model shoulder joint kinematics," Orthopaedics \& Traumatology: Surgery \& Research, 100(7), pp. 715-719.

[44] Klous, M., and Klous, S., 2010, "Marker-based reconstruction of the kinematics of a chain of segments: a new method that incorporates joint kinematic constraints," Journal of Biomechanical Engineering, 132(7), pp. 074501-074501. 
[45] Lu, T. W., and O'Connor, J. J., 1999, "Bone position estimation from skin marker co-ordinates using global optimisation with joint constraints," Journal of Biomechanics, 32(2), pp. 129-134.

[46] Moniz-Pereira, V., Cabral, S., Carnide, F., and Veloso, A. P., 2014, "Sensitivity of joint kinematics and kinetics to different pose estimation algorithms and joint constraints in the elderly," Journal of Applied Biomechanics, 30(3), pp. 446-460.

[47] Ojeda, J., Martínez-Reina, J., and Mayo, J., 2014, "A method to evaluate human skeletal models using marker residuals and global optimization," Mechanism and Machine Theory, 73, pp. 259-272.

[48] Roux, E., Bouilland, S., Godillon-Maquinghen, A. P., and Bouttens, D., 2002, "Evaluation of the global optimisation method within the upper limb kinematics analysis," Journal of Biomechanics, 35(9), pp. 1279-1283.

[49] Stagni, R., Fantozzi, S., and Cappello, A., 2009, "Double calibration vs. global optimisation: performance and effectiveness for clinical application," Gait \& Posture, 29(1), pp. 119-122.

[50] Ausejo, S., Suescun, Á., and Celigüeta, J., 2011, "An optimization method for overdetermined kinematic problems formulated with natural coordinates," Multibody System Dynamics, 26(4), pp. 397-410.

[51] Clément, J., Dumas, R., Hagemeister, N., and de Guise, J. A., 2015, "Soft tissue artifact compensation in knee kinematics by multi-body optimization: Performance of subject-specific knee joint models," Journal of Biomechanics, 48(14), pp. 3796-3802.

[52] Duprey, S., Cheze, L., and Dumas, R., 2010, "Influence of joint constraints on lower limb kinematics estimation from skin markers using global optimization," Journal of Biomechanics, 43(14), pp. 2858-2862.

[53] El Habachi, A., Duprey, S., Cheze, L., and Dumas, R., 2015, "A parallel mechanism of the shoulder - application to multi-body optimisation," Multibody System Dynamics, 33(4), pp. 439-451.

[54] Gasparutto, X., Sancisi, N., Jacquelin, E., Parenti-Castelli, V., and Dumas, R., 2015, "Validation of a multi-body optimization with knee kinematic models including ligament constraints," Journal of Biomechanics, 48(6), pp. 1141-1146.

[55] Clément, J., Dumas, R., Hagemeister, N., and de Guise, J. A., 2017, "Can generic knee joint models improve the measurement of osteoarthritic knee kinematics during squatting activity?," Computer Methods in Biomechanics and Biomedical Engineering, 20(1), pp. 94-103.

[56] Sancisi, N., Gasparutto, X., Parenti-Castelli, V., and Dumas, R., 2017, "A multi-body optimization framework with a knee kinematic model including articular contacts and ligaments," Meccanica, 52(3), pp. 695-711.

[57] Richard, V., Lamberto, G., Lu, T.-W., Cappozzo, A., and Dumas, R., 2016, "Knee kinematics estimation using multi-body optimisation embedding a knee joint stiffness matrix: a feasibility study," PLoS ONE, 11(6), p. e0157010.

[58] Lee, J., Flashner, H., and McNitt-Gray, J. L., 2010, "Estimation of multibody kinematics using position measurements," Journal of Computational and Nonlinear Dynamics, 6(3), pp. 031001-031001.

[59] Koning, B. H. W., van der Krogt, M. M., Baten, C. T. M., and Koopman, B. F. J. M., 2015, "Driving a musculoskeletal model with inertial and magnetic measurement 
units," Computer Methods in Biomechanics and Biomedical Engineering, 18(9), pp. 10031013.

[60] Lund, M. E., Andersen, M. S., de Zee, M., and Rasmussen, J., 2015, "Scaling of musculoskeletal models from static and dynamic trials," International Biomechanics, 2(1), pp. 1-11.

[61] Ojeda, J., Martínez-Reina, J., and Mayo, J., 2016, "The effect of kinematic constraints in the inverse dynamics problem in biomechanics," Multibody System Dynamics, 37(3), pp. 291-309.

[62] Andersen, M. S., Damsgaard, M., MacWilliams, B., and Rasmussen, J., 2010, "A computationally efficient optimisation-based method for parameter identification of kinematically determinate and over-determinate biomechanical systems," Computer Methods in Biomechanics and Biomedical Engineering, 13(2), pp. 171-183.

[63] Lisco, G., Pastorelli, S., and Gastaldi, L., 2016, "Application of a functional method for subject and motion specific joints kinematics during walking " International Journal of Applied Engineering Research, 11(11), pp. 7588-7591.

[64] Debril, J.-F., Pudlo, P., Simoneau, E., Gorce, P., and Lepoutre, F. X., 2011, "A method for calculating the joint coordinates of paraplegic subjects during the transfer movement despite the loss of reflective markers," International Journal of Industrial Ergonomics, 41(2), pp. 153-166.

[65] Sholukha, V., Bonnechere, B., Salvia, P., Moiseev, F., Rooze, M., and Van Sint Jan, S., 2013, "Model-based approach for human kinematics reconstruction from markerless and marker-based motion analysis systems," Journal of Biomechanics, 46(14), pp. 2363-2371.

[66] Marra, M. A., Vanheule, V., Fluit, R., Koopman, B. H. F. J. M., Rasmussen, J., Verdonschot, N., and Andersen, M. S., 2015, "A subject-specific musculoskeletal modeling framework to predict in vivo mechanics of total knee arthroplasty," Journal of Biomechanical Engineering, 137(2), pp. 020904-020904.

[67] Martelli, S., Kersh, M. E., and Pandy, M. G., 2015, "Sensitivity of femoral strain calculations to anatomical scaling errors in musculoskeletal models of movement," Journal of Biomechanics, 48(13), pp. 3615-3624.

[68] Martelli, S., Valente, G., Viceconti, M., and Taddei, F., 2015, "Sensitivity of a subject-specific musculoskeletal model to the uncertainties on the joint axes location," Computer Methods in Biomechanics and Biomedical Engineering, 18(14), pp. 1555-1563.

[69] Reinbolt, J. A., Haftka, R. T., Chmielewski, T. L., and Fregly, B. J., 2007, "Are patient-specific joint and inertial parameters necessary for accurate inverse dynamics analyses of gait?," IEEE Transactions on Biomedical Engineering, 54(5), pp. 782-793.

[70] Valente, G., Pitto, L., Stagni, R., and Taddei, F., 2015, "Effect of lower-limb joint models on subject-specific musculoskeletal models and simulations of daily motor activities," Journal of Biomechanics, 48(16), pp. 4198-4205.

[71] Groen, B. E., Geurts, M., Nienhuis, B., and Duysens, J., 2012, "Sensitivity of the OLGA and VCM models to erroneous marker placement: effects on 3D-gait kinematics," Gait \& Posture, 35(3), pp. 517-521.

[72] El Habachi, A., Moissenet, F., Duprey, S., Cheze, L., and Dumas, R., 2015, "Global sensitivity analysis of the joint kinematics during gait to the parameters of a lower limb multi-body model," Medical \& Biological Engineering \& Computing, 53(7), pp. 655667. 
Journal of Biomechanical Engineering

[73] Pontonnier, C., and Dumont, G., 2010, "From motion capture to muscle forces in the human elbow aimed at improving the ergonomics of workstations," Virtual and Physical Prototyping, 5(3), pp. 113-122.

[74] Bonnechère, B., Sholukha, V., Salvia, P., Rooze, M., and Van Sint Jan, S., 2015, "Physiologically corrected coupled motion during gait analysis using a model-based approach," Gait \& Posture, 41(1), pp. 319-322.

[75] Kainz, H., Modenese, L., Lloyd, D. G., Maine, S., Walsh, H. P. J., and Carty, C. P., 2016, "Joint kinematic calculation based on clinical direct kinematic versus inverse kinematic gait models," Journal of Biomechanics, 49(9), pp. 1658-1669.

[76] Li, K., Zheng, L., Tashman, S., and Zhang, X., 2012, "The inaccuracy of surface-measured model-derived tibiofemoral kinematics," Journal of Biomechanics, 45(15), pp. 2719-2723.

[77] Myers, C. A., Laz, P. J., Shelburne, K. B., and Davidson, B. S., 2015, "A probabilistic approach to quantify the impact of uncertainty propagation in musculoskeletal simulations," Annals of Biomedical Engineering, 43(5), pp. 1098-1111.

[78] Scheys, L., Desloovere, K., Spaepen, A., Suetens, P., and Jonkers, I., 2011, "Calculating gait kinematics using MR-based kinematic models," Gait \& Posture, 33(2), pp. $158-164$.

[79] Sholukha, V., Leardini, A., Salvia, P., Rooze, M., and Van Sint Jan, S., 2006, "Double-step registration of in vivo stereophotogrammetry with both in vitro 6DOFs electrogoniometry and CT medical imaging," Journal of Biomechanics, 39(11), pp. 2087-2095.

[80] Zheng, L., Li, K., Shetye, S., and Zhang, X., 2014, "Integrating dynamic stereo-radiography and surface-based motion data for subject-specific musculoskeletal dynamic modeling," Journal of Biomechanics, 47(12), pp. 3217-3221.

[81] Andersen, M. S., Benoit, D. L., Damsgaard, M., Ramsey, D. K., and Rasmussen, J., 2010, "Do kinematic models reduce the effects of soft tissue artefacts in skin marker-based motion analysis? An in vivo study of knee kinematics," Journal of Biomechanics, 43(2), pp. 268-273.

[82] Ehrig, R. M., Taylor, W. R., Duda, G. N., and Heller, M. O., 2006, "A survey of formal methods for determining the centre of rotation of ball joints," Journal of Biomechanics, 39(15), pp. 2798-2809.

[83] Ehrig, R. M., Taylor, W. R., Duda, G. N., and Heller, M. O., 2007, "A survey of formal methods for determining functional joint axes," Journal of Biomechanics, 40(10), pp. 2150-2157.

[84] Mokhtarzadeh, H., Perraton, L., Fok, L., Muñoz, M. A., Clark, R., Pivonka, P., and Bryant, A. L., 2014, "A comparison of optimisation methods and knee joint degrees of freedom on muscle force predictions during single-leg hop landings," Journal of Biomechanics, 47(12), pp. 2863-2868.

[85] Valente, G., Pitto, L., Testi, D., Seth, A., Delp, S. L., Stagni, R., Viceconti, M., and Taddei, F., 2014, "Are subject-specific musculoskeletal models robust to the uncertainties in parameter identification?," PLoS ONE, 9(11), p. e112625.

[86] Kalman, R. E., 1960, "A new approach to linear filtering and prediction problems," Journal of Basic Engineering, 82(1), pp. 35-45.

Sons, Inc.

[87] Haykin, S., 2001, Kalman filtering and neural networks, John Wiley \& 
[88] Kun, L., Inoue, Y., Shibata, K., and Enguo, C., 2011, "Ambulatory estimation of knee-joint kinematics in anatomical coordinate system using accelerometers and magnetometers," IEEE Transactions on Biomedical Engineering, 58(2), pp. 435-442.

[89] Bélaise, C., Blache, Y., Thouzé, A., Monnet, T., and Begon, M., 2016, "Effect of wobbling mass modeling on joint dynamics during human movements with impacts," Multibody System Dynamics, 38(4), pp. 345-366.

[90] Pain, M. T., and Challis, J. H., 2006, "The influence of soft tissue movement on ground reaction forces, joint torques and joint reaction forces in drop landings," Journal of Biomechanics, 39(1), pp. 119-124.

[91] Halvorsen, K., Söderström, T., Stokes, V., and Lanshammar, H., 2004, "Using an extended Kalman filter for rigid body pose estimation," Journal of Biomechanical Engineering, 127(3), pp. 475-483.

[92] Tondu, B., 2007, "Estimating shoulder-complex mobility," Applied Bionics and Biomechanics, 4(1), pp. 104-112.

[93] Yang, J., Feng, X., Kim, J. H., and Rajulu, S., 2010, "Review of biomechanical models for human shoulder complex," International Journal of Human Factors Modelling and Simulation, 1(3), pp. 271-293.

[94] Benoit, D. L., Damsgaard, M., and Andersen, M. S., 2015, "Surface marker cluster translation, rotation, scaling and deformation: Their contribution to soft tissue artefact and impact on knee joint kinematics," Journal of Biomechanics, 48(10), pp. 21242129.

[95] Dumas, R., Camomilla, V., Bonci, T., Chèze, L., and Cappozzo, A., 2015, "What portion of the soft tissue artefact requires compensation when estimating joint kinematics?," Journal of Biomechanical Engineering, 137(6), pp. 064502-064502.

[96] Bonci, T., Camomilla, V., Dumas, R., Chèze, L., and Cappozzo, A., 2014, "A soft tissue artefact model driven by proximal and distal joint kinematics," Journal of Biomechanics, 47(10), pp. 2354-2361.

[97] Camomilla, V., Bonci, T., Dumas, R., Chèze, L., and Cappozzo, A., 2015, "A model of the soft tissue artefact rigid component," Journal of Biomechanics, 48(10), pp. 1752-1759.

[98] Michaud, B., Jackson, M., Arndt, A., Lundberg, A., and Begon, M., 2016, "Determining in vivo sternoclavicular, acromioclavicular and glenohumeral joint centre locations from skin markers, CT-scans and intracortical pins: A comparison study," Medical Engineering and Physics, 38(3), pp. 290-296.

[99] Pataky, T. C., 2012, "One-dimensional statistical parametric mapping in Python," Computer Methods in Biomechanics and Biomedical Engineering, 15(3), pp. 295 301.

[100] Cereatti, A., Bonci, T., Akbarshahi, M., Aminian, K., Barre, A., Begon, M., Benoit, D. L., Charbonnier, C., Dal Maso, F., Fantozzi, S., Lin, C. C., Lu, T. W., Pandy, M. G., Stagni, R., van den Bogert, A. J., and Camomilla, V., 2017, "Standardization proposal of soft tissue artefact description for data sharing in human motion measurements," Journal of Biomechanics.

[101] Bland, J., and Altman, D. G., 1986, "Statistical methods for assessing agreement between two methods of clinical measurement," The Lancet, 327(8476), pp. 307-310. 
[102] Cleather, D. J., and Bull, A. M. J., 2011, "Knee and hip joint forces sensitivity to the degrees of freedom classification at the knee," Proceedings of the Institution of Mechanical Engineers, Part H: Journal of Engineering in Medicine, 225(6), pp. 621-626.

[103] Dumas, R., Moissenet, F., Gasparutto, X., and Cheze, L., 2012, "Influence of joint models on lower-limb musculo-tendon forces and three-dimensional joint reaction forces during gait," Proceedings of the Institution of Mechanical Engineers, Part H: Journal of Engineering in Medicine, 226(2), pp. 146-160.

[104] Glitsch, U., and Baumann, W., 1997, "The three-dimensional determination of internal loads in the lower extremity," Journal of Biomechanics, 30(11), pp. 1123-1131.

[105] Hicks, J. L., Uchida, T. K., Seth, A., Rajagopal, A., and Delp, S. L., 2015, "Is my model good enough? Best practices for verification and validation of musculoskeletal models and simulations of movement," Journal of Biomechanical Engineering, 137(2), p. 020905.

[106] Lund, M. E., de Zee, M., Andersen, M. S., and Rasmussen, J., 2012, "On validation of multibody musculoskeletal models," Proceedings of the Institution of Mechanical Engineers, Part H: Journal of Engineering in Medicine, 226(2), pp. 82-94. 


\section{Table Caption List}

Table $1 \quad$ Design variables of the MKO problems

Table 2 Constraints in the MKO problems

Table 3 Types of validation of the MKO. Subcategories are explained in the notes $(*, \dagger, \dagger)$

Table 4 Fields of MKO applications. Subcategories are explained in the notes $(*, \dagger, \ddagger, \S,,, ’)$

Table S1 Quality assessment (each question was rated from 0 to 2 or indicated non-applicable): Q1. Are the research objectives clearly stated? Q2. Is the study design clearly described? Q3. Are the optimization method principles clearly explained? Q4. Are implementation details provided? Q5. Were participant characteristics adequately described? Q6. Were movement tasks clearly defined? Q7. Was equipment design and set up clearly described? Q8. Were the evaluation strategy and the reference used appropriately justified? Q9. Were the analytical techniques clearly described? Q10. Were the statistical methods justified and appropriately described? Q11. Were direct results easily interpretable? Q12. Were the main outcomes clearly stated and supported by the results? Q13. Were limitations of the study clearly described? Q14. Were key findings positioned with respect to the state-ofthe-art? Q15. Were conclusions drawn from the study clearly stated? 
Journal of Biomechanical Engineering

\section{Appendix 1}

Table S1

\begin{tabular}{|c|c|c|c|c|c|c|c|c|c|c|c|c|c|c|c|}
\hline \multirow[t]{2}{*}{ Article } & \multicolumn{15}{|c|}{ Questions } \\
\hline & 1 & 2 & 3 & 4 & 5 & 6 & 7 & 8 & 9 & 10 & 11 & 12 & 13 & 14 & 15 \\
\hline Aguiar et al. (2014) [42] & 1 & 2 & 2 & 2 & 2 & 2 & 2 & 0 & 2 & / & 1 & 1 & 0 & 1 & 1 \\
\hline Aguiar et al. (2016) [41] & 2 & 2 & 2 & 1 & 2 & 2 & 1 & 1 & 2 & 2 & 2 & 1 & 0 & 1 & 1 \\
\hline Andersen et al. (2009) [32] & 1 & 1 & 2 & 2 & / & 2 & / & 2 & 2 & / & 1 & 1 & 2 & 1 & 1 \\
\hline Andersen et al. (2010) [81] & 2 & 2 & 2 & 1 & 2 & 2 & 2 & 2 & 2 & 1 & 2 & 2 & 1 & 2 & 2 \\
\hline Andersen et al. (2010) [62] & 1 & 1 & 2 & 2 & 0 & 2 & 2 & 2 & 2 & 1 & 1 & 1 & 1 & 1 & 1 \\
\hline Ausejo et al. (2011) [50] & 2 & 2 & 2 & 2 & 2 & 2 & 2 & 1 & 1 & / & 2 & 1 & 0 & 0 & 1 \\
\hline Ayusawa et al. (2014) [14] & 2 & 2 & 2 & 2 & 0 & 1 & 2 & 1 & 1 & / & 1 & 1 & 0 & 0 & 1 \\
\hline Begon et al. (2008) [15] & 2 & 2 & 2 & 2 & 1 & 2 & 2 & 2 & 2 & 2 & 2 & 2 & 0 & 2 & 2 \\
\hline Begon et al. (2016) [27] & 2 & 2 & 2 & 1 & 2 & 2 & 2 & 2 & 2 & 2 & 2 & 2 & 2 & 2 & 2 \\
\hline Bolsterlee et al. (2014) [12] & 2 & 2 & 2 & 2 & 2 & 1 & 1 & 2 & 2 & 1 & 2 & 2 & 2 & 1 & 2 \\
\hline Bonnechère et al. (2015) [74] & 2 & 2 & 0 & 1 & 2 & 2 & 2 & 0 & 2 & 2 & 2 & 2 & 1 & 1 & 2 \\
\hline Cerveri et al. (2003) [35] & 2 & 2 & 2 & 2 & 1 & 1 & 2 & 2 & 2 & / & 1 & 2 & 0 & 0 & 2 \\
\hline Cerveri et al. (2003) [33] & 2 & 1 & 2 & 2 & 1 & 1 & 2 & 0 & 2 & / & 1 & 2 & 1 & 2 & 2 \\
\hline Cerveri et al. (2005) [34] & 2 & 2 & 2 & 2 & / & 0 & / & 2 & 2 & 2 & 2 & 2 & 0 & 1 & 1 \\
\hline Charbonnier et al. (2014) [43] & 2 & 2 & 2 & 1 & 2 & 2 & 2 & 2 & 2 & / & 2 & 2 & 1 & 2 & 2 \\
\hline Charlton et al. (2004) [16] & 1 & 2 & 2 & 2 & 0 & 1 & 2 & 2 & 2 & 1 & 2 & 2 & 1 & 2 & 2 \\
\hline Clément et al. (2015) [51] & 2 & 2 & 2 & 2 & 2 & 2 & 2 & 2 & 2 & 1 & 2 & 2 & 2 & 2 & 2 \\
\hline Clément et al. (2017) [55] & 2 & 2 & 0 & 1 & 2 & 2 & 2 & 2 & 1 & 2 & 2 & 2 & 2 & 2 & 2 \\
\hline Debril et al. (2011) [64] & 2 & 2 & 2 & 1 & 2 & 2 & 2 & 2 & 2 & 1 & 2 & 2 & 1 & 1 & 2 \\
\hline De Groote et al. (2008) [40] & 2 & 2 & 2 & 1 & 1 & 2 & 1 & 1 & 1 & / & 2 & 2 & 2 & 2 & 2 \\
\hline Duprey et al. (2010) [52] & 2 & 2 & 2 & 2 & 2 & 0 & 1 & 2 & 1 & / & 2 & 2 & 2 & 1 & 2 \\
\hline El Habachi et al. (2015) [53] & 2 & 2 & 2 & 2 & 2 & 2 & 2 & 2 & 2 & 1 & 2 & 2 & 2 & 2 & 2 \\
\hline El Habachi et al. (2015) [72] & 2 & 2 & 2 & 1 & 2 & 2 & 1 & 2 & 2 & 1 & 2 & 2 & 2 & 1 & 1 \\
\hline El-Gohary \& McNames (2012) [36] & 1 & 2 & 2 & 2 & 1 & 2 & 2 & 2 & 1 & 2 & 2 & 2 & 0 & 1 & 2 \\
\hline Fohanno et al. (2013) [17] & 2 & 2 & 1 & 1 & 2 & 2 & 2 & 1 & 2 & 2 & 2 & 2 & 0 & 2 & 2 \\
\hline Fohanno et al. (2014) [11] & 2 & 2 & 2 & 1 & 2 & 2 & 2 & 2 & 2 & 2 & 1 & 2 & 1 & 2 & 2 \\
\hline Gasparutto et al. (2015) [54] & 2 & 2 & 1 & 0 & 2 & 2 & 2 & 2 & 1 & / & 2 & 2 & 2 & 2 & 2 \\
\hline Groen et al. (2012) [71] & 2 & 2 & 2 & 1 & 2 & 1 & 2 & 1 & 0 & 2 & 2 & 2 & 2 & 2 & 2 \\
\hline Jackson et al. (2012) [18] & 2 & 2 & 1 & 1 & 2 & 2 & 2 & 1 & 1 & 2 & 2 & 2 & 1 & 2 & 1 \\
\hline Kaintz et al. (2016) [75] & 2 & 2 & 1 & 0 & 2 & 2 & 2 & 2 & 1 & 2 & 2 & 2 & 1 & 2 & 2 \\
\hline Klous \& Klous (2010) [44] & 2 & 2 & 2 & 1 & / & 1 & / & / & 2 & 2 & 2 & 2 & 1 & 2 & 2 \\
\hline Koning et al. (2015) [59] & 2 & 2 & 2 & 1 & 2 & 2 & 2 & 2 & 2 & / & 2 & 2 & 2 & 2 & 2 \\
\hline Kun et al. (2011) [88] & 2 & 2 & 1 & 2 & 2 & 2 & 2 & 2 & 2 & / & 2 & 2 & 0 & 0 & 1 \\
\hline Laitenberger et al. (2015) [13] & 2 & 2 & 2 & 2 & 2 & 2 & 2 & 2 & 2 & / & 2 & 2 & 2 & 2 & 2 \\
\hline Lamberto et al. (2016) [28] & 2 & 2 & 1 & 1 & 2 & 2 & 2 & 2 & 2 & 2 & 2 & 2 & 2 & 2 & 1 \\
\hline Lathrop et al. (2011) [31] & 2 & 2 & 2 & 2 & 1 & 1 & 2 & 2 & 1 & / & 2 & 2 & 2 & 2 & 2 \\
\hline Lee et al. (2010) [58] & 2 & 2 & 2 & 2 & 0 & 1 & 0 & 0 & 2 & / & 1 & 1 & 0 & 0 & 1 \\
\hline Li et al. (2012) [76] & 2 & 2 & 2 & 1 & 2 & 2 & 2 & 2 & 2 & 2 & 2 & 2 & 2 & 2 & 1 \\
\hline
\end{tabular}


Journal of Biomechanical Engineering

\begin{tabular}{|c|c|c|c|c|c|c|c|c|c|c|c|c|c|c|c|}
\hline \multirow[t]{2}{*}{ Article (continued) } & \multicolumn{15}{|c|}{ Questions } \\
\hline & 12 & & & 4 & 5 & 6 & 7 & 8 & 9 & 10 & 11 & 12 & 13 & & 15 \\
\hline Lisco et al. (2016) [63] & 1 & 2 & 2 & 1 & 1 & 2 & 2 & 1 & 0 & 2 & 2 & 1 & 0 & 0 & 1 \\
\hline Lu \& O’Connor (1999) [45] & 2 & 2 & 2 & 2 & 0 & 0 & 1 & 1 & 1 & / & 2 & 2 & 0 & 0 & 1 \\
\hline Lund et al. (2015) [60] & 2 & 2 & 2 & 1 & 2 & 1 & 1 & 2 & 2 & / & 2 & 2 & 1 & 2 & 2 \\
\hline Mantovani \& Lamontagne (2017) [29] & 2 & 2 & 2 & 1 & 2 & 2 & 2 & 2 & 0 & 2 & 2 & 2 & 2 & 2 & 2 \\
\hline Marra et al. (2015) [66] & 2 & 2 & 1 & 1 & 2 & 2 & $/$ & 2 & 2 & / & 2 & 2 & 2 & 2 & 2 \\
\hline Martelli et al. (2015) [68] & 2 & 2 & 1 & 1 & 2 & 1 & 1 & 1 & 1 & / & 2 & 2 & 2 & 2 & 2 \\
\hline Martelli et al. (2015) [67] & 2 & 2 & 1 & 1 & 2 & 2 & 2 & 2 & 1 & 2 & 2 & 2 & 2 & 2 & 2 \\
\hline Miezal et al. (2016) [37] & 1 & 1 & 2 & 1 & 2 & 1 & 2 & 1 & 1 & / & 1 & 2 & 0 & 1 & 1 \\
\hline Mokhtarzadeh et al. (2014) [84] & 2 & 2 & 1 & 2 & 2 & 2 & 2 & 1 & 2 & 2 & 2 & 2 & 2 & 2 & 2 \\
\hline Moniz-Pereira et al. (2014) [46] & 2 & 2 & 0 & 1 & 1 & 2 & 2 & 1 & 1 & / & 2 & 2 & 2 & 2 & 2 \\
\hline Myers et al. (2015) [77] & 2 & 2 & 2 & 0 & 2 & 2 & 1 & 1 & 2 & 1 & 2 & 2 & 2 & 2 & 2 \\
\hline Ojeda et al. (2014) [47] & 2 & 2 & 2 & 2 & 1 & 1 & 1 & 2 & 2 & / & 2 & 1 & 2 & 1 & 2 \\
\hline Ojeda et al. (2016) [61] & 2 & 2 & 2 & 2 & 1 & 1 & 2 & 2 & 1 & / & 2 & 2 & 0 & 2 & 2 \\
\hline Pizzolato et al. (2017) [30] & 2 & 2 & 1 & 2 & 2 & 2 & 1 & 1 & 1 & / & 2 & 2 & 1 & 2 & 2 \\
\hline Pontonnier \& Dumont (2009) [19] & 2 & 2 & 2 & 2 & 0 & 1 & 0 & / & 1 & / & 2 & 1 & 0 & 1 & 1 \\
\hline Pontonnier \& Dumont (2010) [73] & 1 & 2 & 2 & 2 & 0 & 1 & 2 & / & 2 & / & 2 & 1 & 1 & 1 & 1 \\
\hline Prinold \& Bull (2014) [20] & 1 & 2 & 2 & 2 & 1 & 1 & 1 & 1 & 2 & 2 & 2 & 2 & 0 & 2 & 2 \\
\hline Prokopenko et al. (2001) [21] & 2 & 2 & 2 & 2 & 2 & 2 & 2 & 2 & 2 & 2 & 1 & 2 & 0 & 2 & 2 \\
\hline Reinbolt et al. (2005) [22] & 0 & 2 & 2 & 2 & 0 & 1 & 1 & 2 & 2 & / & 2 & 2 & 2 & 2 & 2 \\
\hline Reinbolt et al. (2007) [69] & 0 & 2 & 2 & 2 & 2 & 2 & 1 & 2 & 2 & 2 & 2 & 2 & 1 & 1 & 2 \\
\hline Richard et al. (2016) [57] & 2 & 2 & 2 & 2 & 2 & 2 & 2 & 2 & 2 & 2 & 2 & 2 & 2 & 2 & 2 \\
\hline Robinson et al. (2014) [23] & 2 & 2 & 2 & 2 & 2 & 2 & 2 & 2 & 1 & 2 & 2 & 2 & 2 & 2 & 2 \\
\hline Roux et al. (2002) [48] & 2 & 2 & 1 & 2 & / & 2 & $/$ & 2 & 2 & / & 2 & 2 & 0 & 1 & 2 \\
\hline Sancisi et al. (2017) [56] & 2 & 1 & 2 & 2 & 1 & 2 & 1 & 1 & 2 & / & 1 & 1 & 1 & 2 & 2 \\
\hline Scheys et al. (2011) [78] & 2 & 2 & 2 & 1 & 2 & 2 & 2 & 1 & 2 & 2 & 2 & 2 & 1 & 1 & 2 \\
\hline Seth et al. (2016) [39] & 2 & 2 & 2 & 2 & 1 & 2 & 1 & 2 & 2 & / & 2 & 2 & 2 & 1 & 2 \\
\hline Sholukha et a. (2006) [79] & 0 & 2 & 2 & 2 & 2 & 2 & 2 & 2 & 2 & 1 & 1 & 2 & 1 & 2 & 1 \\
\hline Sholukha et al. (2013) [65] & 1 & 1 & 1 & 0 & 1 & 1 & 1 & 0 & 1 & / & 1 & 1 & 1 & 2 & 1 \\
\hline Stagni et al. (2009) [49] & 2 & 2 & 1 & 1 & 2 & 2 & 1 & 2 & 1 & 2 & 2 & 2 & 0 & 2 & 2 \\
\hline Thouzé et al. (2016) [24] & 2 & 2 & 2 & 1 & 2 & 2 & 2 & 1 & 2 & 2 & 2 & 2 & 2 & 2 & 2 \\
\hline Tsai \& Lung (2014) [26] & 2 & 2 & 2 & 2 & 0 & 0 & 1 & 0 & 2 & / & 1 & 2 & 0 & 0 & 2 \\
\hline Valente et al. (2014) [85] & 2 & 2 & 1 & 1 & 2 & 2 & 2 & 2 & 1 & 2 & 2 & 2 & 2 & 1 & 2 \\
\hline Valente et al. (2015) [70] & 2 & 2 & 1 & 1 & 2 & 2 & 2 & 2 & 1 & 2 & 2 & 2 & 2 & 2 & 2 \\
\hline van den Bogert et al. (2013) [25] & 2 & 2 & 2 & 2 & 2 & 2 & 2 & 0 & 2 & / & 2 & 2 & 1 & 2 & 2 \\
\hline Zhang et al. (2011) [38] & 0 & 2 & 2 & 2 & 0 & 2 & 2 & 2 & 1 & / & 2 & 2 & 0 & 1 & 2 \\
\hline Zheng et al. (2014) [80] & 2 & 2 & 1 & 1 & 1 & 2 & 2 & 2 & 1 & / & 2 & 2 & 2 & 1 & 1 \\
\hline
\end{tabular}

